\title{
Modified VMAT Plans for Locally Advanced Centrally Located Non-Small Cell Lung Cancer (NSCLC)
}

\author{
Eva Y. W. Cheung ${ }^{1, *}$, Virginia H. Y. Kwong ${ }^{2}$, Fandy Y. C. Chan ${ }^{1}$, Dominic Y. T. Cheng ${ }^{1}$, Janice K. Y. Cheng ${ }^{1}$, \\ Sapphire H. Y. Yung ${ }^{1}$, Kiris T. K. Chan ${ }^{1}$, Kelly T. Y. Cheung ${ }^{1}$, Tracy S. W. Cheung ${ }^{1}$ and Janna C. L. Yiu ${ }^{1}$ \\ 1 School of Medical Health and Sciences, Tung Wah College, 19/F, 31 Wylie Road, Ho Man Tin \\ Hong Kong, China; $17003980 @ t w c . e d u . h k$ (F.Y.C.C.); $18001627 @ t w c . e d u . h k$ (D.Y.T.C.); \\ 17002895@twc.edu.hk (J.K.Y.C.); 17002595@twc.edu.hk (S.H.Y.Y.); 16003178@twc.edu.hk (K.T.K.C.); \\ 16003318@twc.edu.hk (K.T.Y.C.); 16000196@twc.edu.hk (T.S.W.C.); 15000109@twc.edu.hk (J.C.L.Y.) \\ 2 Department of Clinical Oncology, Prince of Wales Hospital, 30-32 Ngan Shing Street, Shatin, New Territories, \\ Hong Kong, China; Khy806@ha.org.hk \\ * Correspondence: evacheung@twc.edu.hk or cheungevayw@gmail.com
}

\section{check for} updates

Citation: Cheung, E.Y.W.; Kwong, V.H.Y.; Chan, F.Y.C.; Cheng, D.Y.T.; Cheng, J.K.Y.; Yung, S.H.Y.; Chan, K.T.K.; Cheung, K.T.Y.; Cheung, T.S.W.; Yiu, J.C.L. Modified VMAT Plans for Locally Advanced Centrally Located Non-Small Cell Lung Cancer (NSCLC). Life 2021, 11, 1085. https:// doi.org/10.3390/life11101085

Academic Editors: Andrew Edet Ekpenyong and Tamer Refaat

Received: 27 August 2021

Accepted: 11 October 2021

Published: 14 October 2021

Publisher's Note: MDPI stays neutral with regard to jurisdictional claims in published maps and institutional affiliations.

Copyright: (c) 2021 by the authors. Licensee MDPI, Basel, Switzerland. This article is an open access article distributed under the terms and conditions of the Creative Commons Attribution (CC BY) license (https:// creativecommons.org/licenses/by/ $4.0 /)$.

\begin{abstract}
Objectives: This study aimed to find the optimal radiotherapy VMAT plans, that achieved high conformity and homogeneity to the planned target volume (PTV), and minimize the dose to nearby organs at risk including the non-PTV lung, heart and oesophagus for patients with centrally located non-small Cell Lung Cancer. Methods: A total of 18 patients who were treated for stage III centrally located non-small Cell Lung Cancer were selected retrospectively for this study. Identical CT datasets, 4D CT and structure dataset were used for radiotherapy planning based on single-planar VMAT (SP-VMAT), dual-planar VMAT (DP-VMAT) and Hybrid VMAT (H-VMAT). For SP-VMAT, one full arc and two half arcs were created on single-plane with couch at $0^{\circ}$. For DP-VMAT, one full arc was created with couch at $0^{\circ}$, and two half arcs with couch rotation of $330^{\circ}$ or $30^{\circ}$. For H-VMAT, anterior-posterior opposing fixed beam and two half arcs were planned at couch at $0^{\circ}$. Dose constraints were adhered to the RTOG0617. Dose volumetric parameters were collected for statistical analysis. Results: There were no significant differences for the PTV, HI, CI between the SP-VMAT, DP-VMAT and H-VMAT. For the non-PTV lungs, Dmean, V20, V10, V5, D1500 and D1000 were significantly lower (2.05 Gy, 6.47\%, 15.89\%, 11.66\% 4.17 Gy and 5.47 Gy respectively) in H-VMAT than that of SP-VMAT (all $p<0.001$ ). For the oesophagus, Dmax, Dmean, V30 and V18.8 of H-VMAT were $0.08 \mathrm{~Gy}, 1.73 \mathrm{~Gy}, 5.54 \%$ and $7.17 \%$ lower than that of the SP-VMAT plan. For the heart, Dmean, V34, V28, V20 and V10 of DP-VMAT were lower than that of SP-VMAT by 1.45 Gy, $0.65 \%$, $1.74 \%, 4.8 \%$ and $7.11 \%$ respectively. Conclusion: The proposed H-VMAT showed more favourable plan quality than the SP-VMAT for centrally located stage III NSCLC, in particular for non-PTV lungs and the oesophagus. It will benefit patients, especially those who planned for immunotherapy (Durvalumab) after standard chemo-irradiation. The proposed DP-VMAT plan showed significant dose reduction to the heart when compared to the H-VMAT plan.
\end{abstract}

Keywords: non-small cell lung cancer (NSCLC); volumetric modulated arc therapy; centrally located; lungs; oesophagus; heart; Durvalumab; immunotherapy; non-PTV lung

\section{Introduction}

Lung cancer is the second commonly diagnosed cancer in the world, with 2.24 million new cases annually. It has been the leading cause of cancer death, with 1.8 million deaths worldwide [1]. In Hong Kong, the incidence rate is the second (15.4\%) among all cancer sites. It has also been ranked as having the highest mortality, of $26.4 \%$, among all cancer sites in 2018 [2].

The standard of care of locally advanced non-small cell lung cancer (NSCLC) is concomitant chemo-irradiation [3]. However, it is known that combining the two treatments increases pulmonary, cardiac and oesophageal toxicities [4-7], and the median 5-year 
progression-free survival rate was only $15 \%[8,9]$. The application of immunotherapy (Durvalumab) after chemo-irradiation in the PACIFIC trial demonstrated 17\% improvement in the 18-month progression-free survival, but introduced Grade 3 or 4 adverse events, including pneumonitis [10]. Due to the systemic nature of chemotherapy and immunotherapy, the role of radiotherapy (RT) to minimize local regional thoracic toxicity was particularly important. In RT, volumetric modulated arc therapy (VMAT) is adopted in recent years to achieve superior conformity and dose escalation to the tumour, at the expense of more volume of nearby organs receiving a low dose. In this case, minimizing the dose to nearby organs at risk (OARs) becomes crucial when the OAR is radiosensitive. In thoracic regions, the low dose to the lungs, heart and oesophagus are major concerns in radiotherapy planning.

Several studies have demonstrated that modification of co-planar VMAT plans may reduce dose to OARs [11-16]. For example, non-coplanar VMAT plans reduced the low dose to the heart during dose delivery, while an ipsilateral lung might expose to higher radiation dose [11]. Hybrid 3DCRT/VMAT (H-VMAT), which includes a pair of AP opposing beams and a pair of partial arcs, is used as a modification of the VMAT plan. It can reduce the dose to the lungs and spinal cord when comparing to co-planar VMAT [16].

The sparing of OARs is challenging when the tumour is centrally located, and/or with lymph node involvement. To deliver the escalated prescribed dose to the target volume, keeping the dose to the lungs, heart and oesophagus less than the tolerance level is also challenging, with the proximity of their location to the target volume. Modified beam angles or arcs entry from different planes can be a solution to disperse the low dose by combining gantry rotation and couch angles appropriately. In this study, through demonstrating different beam and couch configurations, i.e., single-planar VMAT (SP-VMAT), dual-planar VMAT (DP-VMAT) and 3DCRT/VMAT (H-VMAT), and comparing their dose volumetric parameters, it is aimed to explore optimal beam arrangements for stage III centrally located NSCLC patients, to minimize their pulmonary, cardiac and oesophageal toxicities.

\section{Materials and Methodology}

\subsection{Patient Selection}

Patients were selected retrospectively from the Clinical Oncology Department of the Prince of Wales Hospital (PWH), New Territories East Cluster, Hospital Authority, Hong Kong. They were treated for non-small cell lung carcinoma (NSCLC) in 2018-2019. Patients who had been diagnosed with stage III NSCLC, with centrally located tumours in lung, or with mediastinal lymph nodes involvement, whose original radiotherapy treatments were planned on being a free-breathing CT image, were included. Patients who were diagnosed with metastases, received radiotherapy treatment previously or had undergone surgical treatment, were excluded.

Ethics approval was obtained from the Research Ethics Committee of the Hospital Authority, The New Territories East Cluster, Hong Kong SAR (CREC Ref No: CUHK-NTEC 2019.655).

\subsection{Simulation}

Philips Brilliance CT-scanner at PWH was used to perform simulation. Patients were simulated in supine position, with wing board and vacuum vaclok as immobilization devices to support both their arms raised over the head. Slice thickness was set as $3 \mathrm{~mm}$. This is standard setup for lung tumour treatment in PWH. A free-breathing CT image was acquired without a contrast agent. $4 \mathrm{D} \mathrm{CT}$ was also acquired to measure the tumour motion within the breathing cycle. For this cohort, patients had compromised lung function and large tumours. In consideration of patient tolerance and reproducibility, clinical team decided not to restrict breathing motion using motion management devices. Magnetic resonance image (MRI) was acquired to improve OARs contouring. 


\subsection{Image Registration, Target Volume and OAR Contouring}

A total of 18 patients met the above inclusion criteria. Magnetic resonance image (MRI) and free-breathing CT image were co-registered. 4D CT was used to determine the movement of the tumour during the respiratory cycle. The oncologists in $\mathrm{PWH}$ were responsible for contouring the planning target volume (PTV). Dosimetrists or radiation therapists in PWH were responsible for contouring the organs-at-risk (OARs), including both lungs, heart, and spinal cord.

The investigators of this study were responsible for contouring the oesophagus with reference to the online RTOG contouring atlas (https://www.rtog.org/CoreLab/ ContouringAtlases.aspx, accessed on 1 April 2020): "RTOG1106 Atlas for Organs at Risk (OARs) in Thoracic Radiation Therapy Structures". All structures were confirmed by radiation therapists in $\mathrm{PWH}$, and approved by certified medical dosimetrist before planning.

\subsection{Treatment Planning}

All treatment plans were done in the radiotherapy planning laboratory at Tung Wah College. To design plans that were compatible to the clinical setting in $\mathrm{PWH}$, the Varian linear accelerator model 21IX (Varian Medical System, Palo Alto, CA, USA) was chosen for all plans in the software Eclipse Radiotherapy Treatment Planning System (Varian Medical Systems, Palo Alto, CA, USA). The version was 15.6. The machine was equipped with 120 High Definition multileaf collimator (MLC) system, and the motion type was sliding window.

For SP-VMAT, DP-VMAT and the VMAT components of H-VMAT plans, $6 \mathrm{MV}$ photon with dose rate of 600 monitor unit (MU) per minute was chosen. Gantry speed was set at 4.8 degree/second. The isocentre and the field sizes were custom fitted to the PTV $+0.5 \mathrm{~cm}$ for each patient automatically by the arc geometry tool in the software. To offset the inter-leaf transmission, collimator was rotated 30 degrees in clockwise and counter-clockwise arcs.

\subsection{Beam and Couch Configuration}

The SP-VMAT plans were composed of three arcs, including one full arc with gantry rotate from 181 degree to 179 degree, and two half arcs with gantry rotate from 181 degree to 0 degree for a tumour situated on right side of the lung; or two half arcs with gantry rotate from 179 degree to 0 degree for a tumour situated on left side of the lung. Couch was set at 0 degrees for all arcs. Details of SP-VMAT plan are shown in Figure 1.

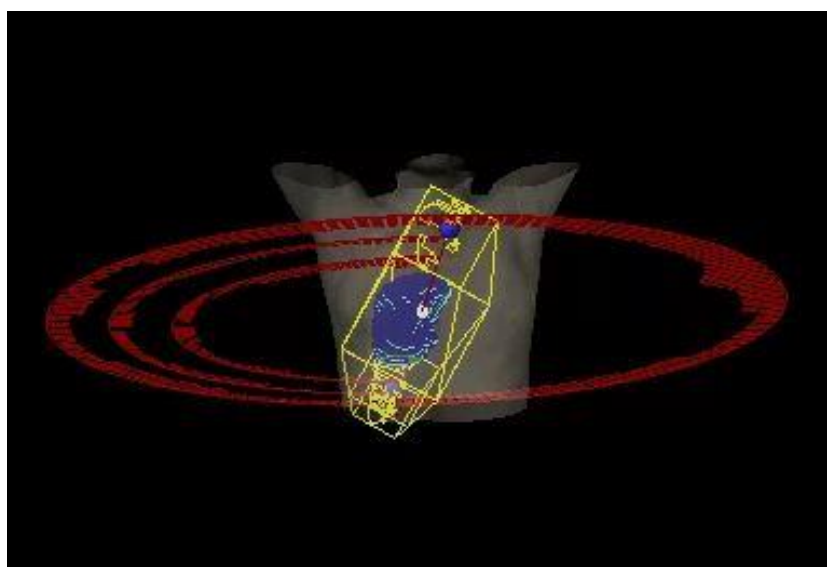

Figure 1. SP-VMAT configuration.

The DP-VMAT plans were composed of three arcs, including one full arc with gantry rotating from 181 degrees to 179 degrees, and two half arcs with gantry rotating from 181 degrees to 0 degrees for a tumour situated on right side of the lung; or two half arcs with gantry rotating from 179 degrees to 0 degrees for a tumour situated on left side of the lung. Couch was set at 0 degrees for the full arc. It was set at 23-30 degrees for the two 
half arcs for a tumour situated on right side of the lung, and 330-337 degrees for a tumour situated on left side of the lung. Details of the DP-VMAT plan are shown in Figure 2.

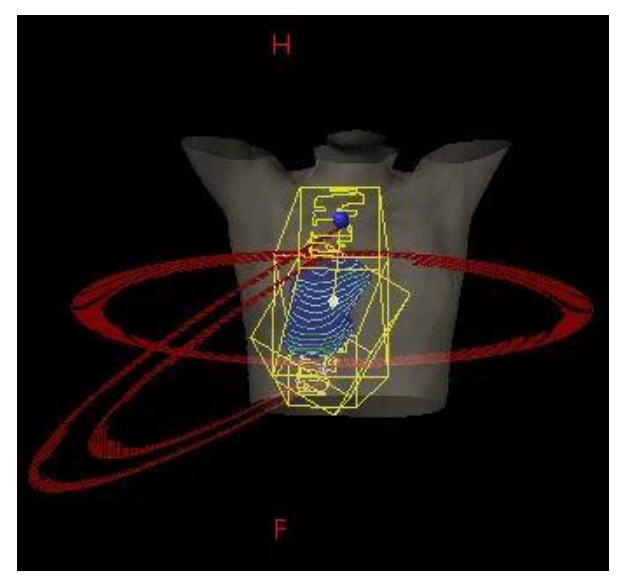

Figure 2. DP-VMAT configuration.

The H-VMAT plans were composed of a pair of anterior-posterior opposing (AP) fields (3DCRT component) and two partial arcs (VMAT component). A $6 \mathrm{MV}$ or $10 \mathrm{MV}$ photon was chosen for AP fields to obtain a balanced dose distribution. The field size was determined by the beams-eye-view (BEV) with MLC fitted to the PTV $+0.5 \mathrm{~cm}$ margin. The gantry angle of the AP fields was 0 degrees and 180 degrees. The VMAT component was composed of two half arcs, with gantry rotating from 181 degrees to 30 degrees for a tumour situated on right side of the lung; or with gantry rotating from 179 degrees to 330 degrees for a tumour situated on left side of the lung. Couch was set at 0 degrees for all arcs. Details of H-VMAT plan was shown in Figure 3.

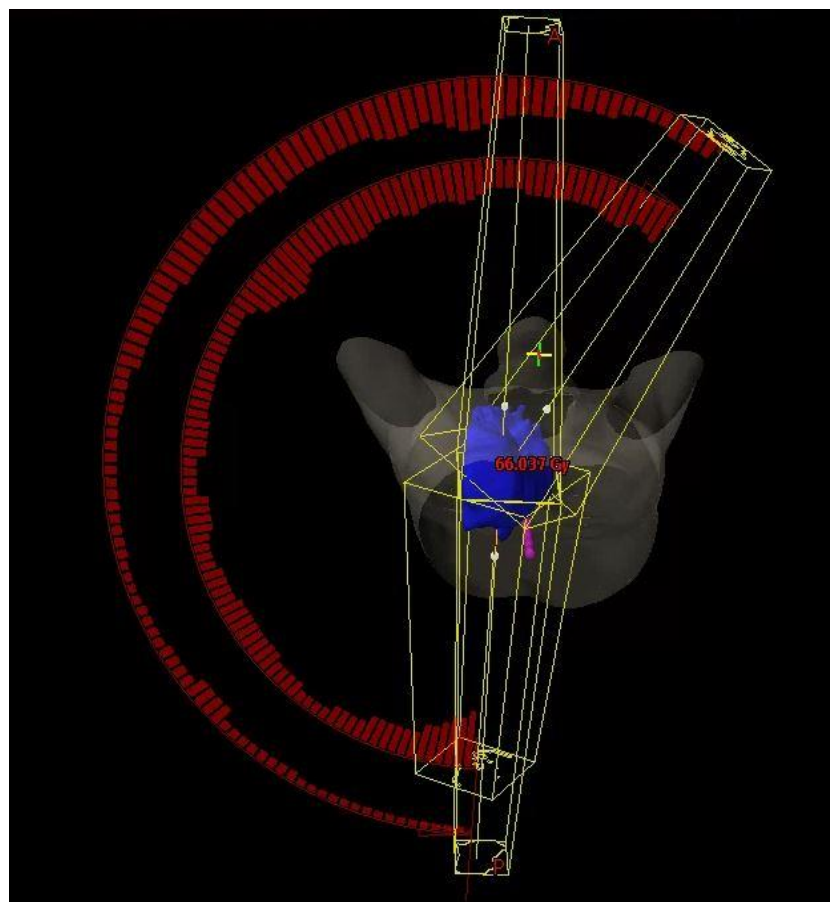

Figure 3. H-VMAT configuration.

\subsection{Dose Prescription}

RTOG 0617 standard dose regime of $60 \mathrm{~Gy}$ was adopted [17]. For SP-VMAT and DP-VMAT, dose prescription was 2 Gray (Gy) per fraction ( $2 \mathrm{~Gy} / \mathrm{fr}$ ), five fractions per 
week for 30 fractions to a total of 60 Gy. For H-VMAT, 50\% of prescribed dose (30 Gy) was allotted to AP fields, which was set as the base plan. The rest of $50 \%$ prescribed dose (30 Gy) was allotted to the VMAT for intensity modulation. Altogether, 60 Gy prescribed dose was planned for PTV.

\subsection{Plan Optimization}

The progressive resolution optimiser 3 (PRO3), version 15.6 (Varian Medical systems, Palo Alto, CA, USA) was adopted to optimise the SP-VMAT, DP-VMAT and the VMAT component of H-VMAT plans. Dose constraints are listed in Table 1. During the optimisation process, weighting applied for each constraint was identical in all plans, so as to ensure the DV parameters obtained corresponded to the planning techniques, but not due to the effects of weighting. Details of the inverse planning algorithm constraints and weighting are listed in Table 2. Anisotropic Analytical Algorithm (AAA), Version 15.6, (Varian Medical systems, Palo Alto, CA, USA) was adopted to calculate dose distribution with $1.25 \mathrm{~mm}$ grid size after optimisation.

Table 1. Planning Dose constraints.

\begin{tabular}{cc}
\hline Critical Organ/Organ-at Risk & Dose Constraint \\
\hline Spinal Cord & Maximum dose $<45 \mathrm{~Gy}$ \\
Non-PTV Lung & Mean dose $<20 \mathrm{~Gy}$ \\
& V $20<35 \%$ \\
V $5<65 \%$ \\
Oesophagus & Maximum dose $<63 \mathrm{~Gy}(105 \%$ of prescribed dose $)$ \\
& Mean dose $<34$ Gy \\
& Mean dose $<35 \mathrm{~Gy}$ \\
Heart & V40 $<80 \%$ \\
& V $45<60 \%$ \\
& V60 $630 \%$ \\
\hline
\end{tabular}

Table 2. Inverse planning algorithm constraints.

\begin{tabular}{ccc}
\hline Structure & Dose Constraint & Weight \\
\hline PTV & Maximum dose $<60 \mathrm{~Gy}$ & 100 \\
Spinal Cord & Minimum dose $>57 \mathrm{~Gy}$ & 100 \\
Non-PTV lung & Maximum dose $<45 \mathrm{~Gy}$ & 100 \\
Heart & Mean dose $<20 \mathrm{~Gy}$ & 50 \\
Oesophagus & V $20<35 \%$ & 50 \\
\hline
\end{tabular}

\subsection{Inter-Planner Variability}

To minimize the inter-planner variabilities, planners with similar clinical experience were recruited to perform planning. Each planner was responsible for all three plans, including SP-VMAT, DP-VMAT and H-VMAT for the same patient. Planning procedures were standardized and four sets of CT were used randomly as samples. All planners performed planning on these CT sample sets. Dose-volume metrics of the plans were used to determine the planner's performance [18]. They started the planning for this study once they met the specified planning goals of each plan.

\subsection{Evaluation of Treatment Plans}

The following criteria were employed as planning goals of each plan:

1. $100 \%$ of prescribed dose (60 Gy) should cover more than $98 \%$ of PTV

2. Maximum dose of a $2 \%$ of PTV should be lower than $108 \%$ of the prescribed dose (64.8 Gy). 
3. Maximum point dose (i.e., hotspots outside the PTV) should be lower than $108 \%$ of the prescribed dose (64.8 Gy).

4. Dose constraints in Tables 1 and 2 should be met.

To evaluate the plan quality, homogeneity index and conformity index were calculated as follows:

Homogeneity index was calculated as:

$$
\mathrm{HI}=\left(\mathrm{D}_{2 \%}-\mathrm{D}_{98 \%}\right) / \mathrm{DP}
$$

where $\mathrm{D}_{2} \%$ is the dose received by $2 \%$ (volume) of planning target volume (PTV), $\mathrm{D}_{98} \%$ is the dose received by $98 \%$ of PTV and DP is the prescribed dose (60 Gy) [19].

Conformity Index was calculated as:

$$
\mathrm{CI}=\mathrm{TV} / \mathrm{PTV}
$$

where TV is the treated volume enclosed by $100 \%$ isodose level. PTV is the planned target volume. The $\mathrm{CI}$ approach to 1 indicated the dose coverage of target volume conformed more to the PTV [20].

For the PTVs, the maximum dose $\left(D_{\max }\right)$, minimum dose $\left(D_{\min }\right)$, mean dose $\left(\mathrm{D}_{\text {mean }}\right)$, minimum dose for $98 \%$ volume of PTV $\left(\mathrm{D}_{98} \%\right), \mathrm{D}_{95 \%}, \mathrm{D}_{50 \%}$ and $\mathrm{D}_{2 \%}$ were calculated.

For non-PTV lung, ipsilateral lung, contralateral lung and both lungs, $\mathrm{D}_{\text {mean }}, \mathrm{V}_{20 \%}$, $\mathrm{V}_{10 \%}, \mathrm{~V}_{5 \%}, \mathrm{D}_{1500 \mathrm{cc}}, \mathrm{D}_{1000 \mathrm{cc}}$ were calculated. For the heart, $\mathrm{D}_{\max }$ and $\mathrm{D}_{\text {mean, }}, \mathrm{V}_{60 \%}, \mathrm{~V}_{45 \%}$, $\mathrm{V}_{40 \%}, \mathrm{~V}_{20 \%}, \mathrm{~V}_{10 \%}, \mathrm{D}_{0.1 \mathrm{cc}}, \mathrm{D}_{2} \%$ were calculated. For oesophagus, $\mathrm{D}_{\max }$ and $\mathrm{D}_{\text {mean }}, \mathrm{V}_{30 \%}$, $\mathrm{V}_{18.8 \%}$ were calculated. For spinal cord, $\mathrm{D}_{\max }$ and $\mathrm{D}_{0.1 \mathrm{cc}}$ were calculated.

\subsection{Statistical Analysis}

IBM Statistical Product and Service Solutions (SPSS) (Version 25.0) was employed to perform statistical analysis. Non-parametric Wilcoxon signed-rank test was performed to compare the mean of the each dosimetric parameters in SP-VMAT, DP-VMAT and H-VMAT. In this study, $p$ values of less than 0.05 were regarded as statistically significant [21].

Certified medical dosimetrists checked and approved all 54 plans to ensure they were clinically acceptable. The results presented in this study were based on these approved plans.

\section{Result}

\subsection{Patient Demographic}

A total of 18 patients were recruited in this study. Their age ranged from 56 to 84 years old. There were 15 males and three females. All patients were diagnosed with NSCLC, 10 were diagnosed with IIIA, and eight were diagnosed with IIIB. The gross tumour size ranged from $97.8 \mathrm{~cm}^{3}$ to $823.5 \mathrm{~cm}^{3}$, with mean tumour size of $428.76 \mathrm{~cm}^{3}$.

\subsection{Dose-Volumetric of PTV}

The overall HI, CI and dose-volumetric parameters of PTV in SP-VMAT, DP-VMAT and H-VMAT were similar and there were no significant differences. Details are shown in Table 3. The overall dose distributions are shown in Figure 4. 
Table 3. Dose-volumetric parameters of the PTV.

\begin{tabular}{|c|c|c|c|c|c|c|c|}
\hline \multirow{2}{*}{$\begin{array}{c}\text { PTV } \\
\text { DV Parameters }\end{array}$} & \multirow{2}{*}{$\begin{array}{c}\text { SP-VMAT } \\
\text { Mean } \pm \text { S.D. }\end{array}$} & \multirow{2}{*}{$\begin{array}{c}\text { DP-VMAT } \\
\text { Mean } \pm \text { S.D. }\end{array}$} & \multirow{2}{*}{$\begin{array}{c}\text { H-VMAT } \\
\text { Mean } \pm \text { S.D. }\end{array}$} & \multicolumn{2}{|c|}{$\begin{array}{c}\text { (DP-VMAT Minus } \\
\text { SP-VMAT) }\end{array}$} & \multicolumn{2}{|c|}{$\begin{array}{c}\text { (H-VMAT Minus } \\
\text { SP-VMAT) }\end{array}$} \\
\hline & & & & Diff & $p$ & Diff & $p$ \\
\hline Maximum Dose (Gy) & $64.59 \pm 0.67$ & $64.68 \pm 0.81$ & $65.54 \pm 0.57$ & 0.09 & 0.472 & 0.95 & 0.23 \\
\hline Mean Dose (Gy) & $61.49 \pm 0.17$ & $61.45 \pm 0.26$ & $61.71 \pm 0.26$ & -0.04 & 0.446 & 0.23 & 0.22 \\
\hline Minimum Dose (Gy) & $55.25 \pm 1.14$ & $57.15 \pm 1.09$ & $54.26 \pm 2.08$ & 1.92 & 0.43 & 9.01 & 0.60 \\
\hline $\mathrm{D} 98 \%(\mathrm{~Gy})$ & $60.42 \pm 0.34$ & $60.37 \pm 0.34$ & $60.54 \pm 0.26$ & -0.05 & 0.248 & 0.12 & 0.33 \\
\hline D95\% (Gy) & $60.75 \pm 0.21$ & $60.69 \pm 0.24$ & $60.77 \pm 0.27$ & -0.06 & 0.267 & 0.02 & 0.95 \\
\hline D50\% (Gy) & $61.51 \pm 0.17$ & $61.46 \pm 0.28$ & $61.66 \pm 0.32$ & -0.05 & 0.36 & 0.16 & 0.12 \\
\hline $\mathrm{D} 2 \%$ (Gy) & $62.49 \pm 0.244$ & $62.48 \pm 0.36$ & $63.25 \pm 0.35$ & -0.01 & 0.744 & 0.75 & 0.26 \\
\hline Homogeneity Index & $0.0338 \pm 0.006$ & $0.0354 \pm 0.01$ & $0.0447 \pm 0.01$ & 0.0016 & 0.215 & 0.01 & 0.1 \\
\hline Conformity Index & $1.1889 \pm 0.09$ & $1.1894 \pm 0.07$ & $1.2165 \pm 0.089$ & 0.0006 & 0.943 & 0.03 & 0.132 \\
\hline
\end{tabular}

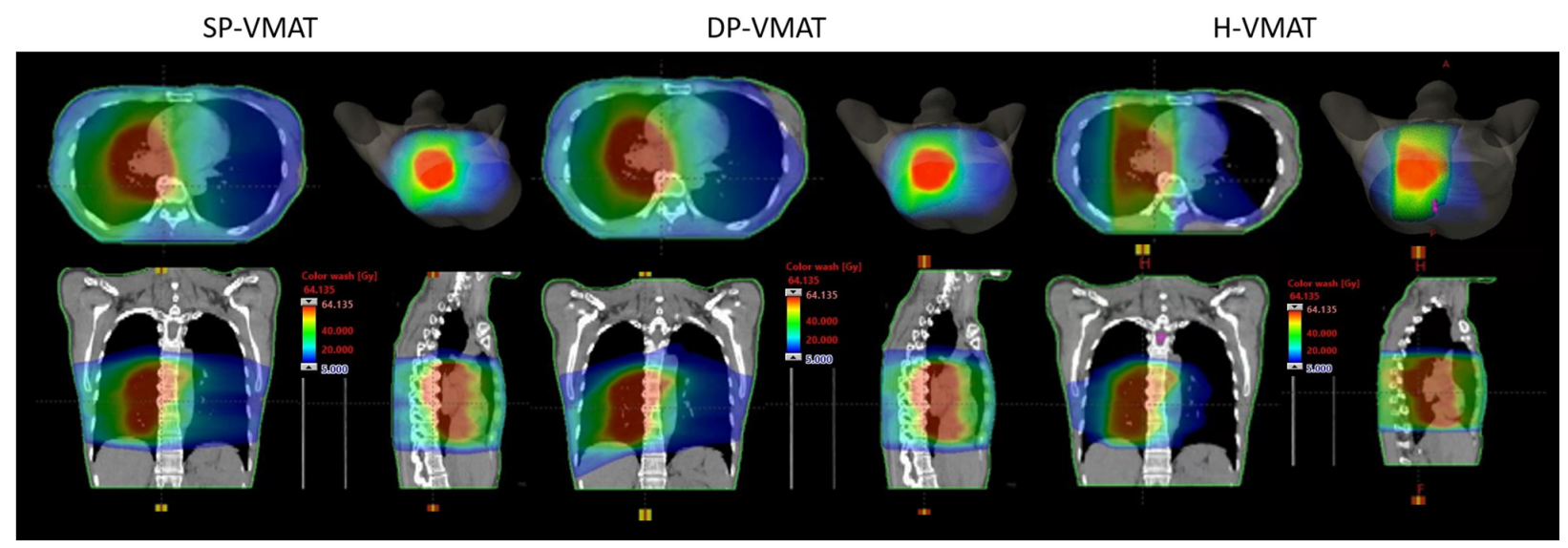

Figure 4. The dose distribution in SP-VMAT, DP-VMAT and H-VMAT in the representative case (case number 8).

\subsection{Dose-Volumetric Parameters of the Lungs}

For non-PTV lungs, the V5 Gy was significantly higher in DP-VMAT when compared to SP-VMAT. All non-PTV lung parameters in H-VMAT were significantly lower than those in SP-VMAT. For ipsilateral lung, all parameters were significantly higher in DP-VMAT when compared to SP-VMAT, but the difference was minimal. For contralateral lung, all parameters were significantly lower in DP-VMAT when compared to SP-VMAT. Consider H-VMAT plans, all parameters were significantly lower in SP-VMAT in both ipsilateral and contralateral lungs. Details of dose-volumetric parameters of the lungs are shown in Table 4.

Table 4. Dose-volumetric parameters of the lungs.

\begin{tabular}{|c|c|c|c|c|c|c|c|c|}
\hline & \multirow{2}{*}{ DV Parameters } & \multirow{2}{*}{$\begin{array}{c}\text { SP-VMAT } \\
\text { Mean } \pm \text { S.D. }\end{array}$} & \multirow{2}{*}{$\begin{array}{c}\text { DP-VMAT } \\
\text { Mean } \pm \text { S.D. }\end{array}$} & \multirow{2}{*}{$\begin{array}{c}\text { H-VMAT } \\
\text { Mean } \pm \text { S.D. }\end{array}$} & \multicolumn{2}{|c|}{$\begin{array}{c}\text { DP-VMAT Minus } \\
\text { SP-VMAT }\end{array}$} & \multicolumn{2}{|c|}{$\begin{array}{l}\text { H-VMAT Minus } \\
\text { SP-VMAT }\end{array}$} \\
\hline & & & & & Diff & $p$ & Diff & $p$ \\
\hline \multirow[t]{6}{*}{$\begin{array}{l}\text { Non-PTV } \\
\text { lung }\end{array}$} & Mean Dose (Gy) & $15.24 \pm 3.18$ & $15.53 \pm 2.76$ & $13.19 \pm 2.47$ & 0.28 & 0.112 & $-2.05 *$ & $<0.0001$ \\
\hline & V20 Gy (\%) & $28.3 \pm 6.98$ & $28.52 \pm 6.97$ & $21.83 \pm 4.26$ & 0.22 & 0.647 & $-6.47^{*}$ & $<0.0001$ \\
\hline & V10 Gy (\%) & $51.73 \pm 12.05$ & $51.46 \pm 9.93$ & $35.84 \pm 6.08$ & -0.26 & 0.695 & $-15.89 *$ & $<0.0001$ \\
\hline & V5 Gy (\%) & $57.95 \pm 14.65$ & $61.41 \pm 11.83$ & $49.29 \pm 9.51$ & $3.45 *$ & 0.006 & $-11.66 *$ & $<0.0001$ \\
\hline & D1500 (Gy) & $10.44 \pm 4.26$ & $10.54 \pm 3.92$ & $6.29 \pm 2.34$ & 0.097 & 0.948 & $-4.17 *$ & $<0.0001$ \\
\hline & D1000 (Gy) & $16.67 \pm 5.34$ & $17.12 \pm 4.89$ & $11.19 \pm 3.88$ & 0.458 & 0.349 & $-5.47 *$ & $<0.0001$ \\
\hline \multirow[t]{6}{*}{ Both Lungs } & Mean Dose (Gy) & $17.31 \pm 3.59$ & $17.58 \pm 3.16$ & $15.31 \pm 2.98$ & 0.27 * & 0.002 & -2.00 & $<0.0001$ \\
\hline & V20 Gy (\%) & $31.51 \pm 7.36$ & $31.73 \pm 7.37$ & $25.23 \pm 5.01$ & 0.22 & 0.231 & $-6.27 *$ & $<0.0001$ \\
\hline & V10 Gy (\%) & $53.87 \pm 12.03$ & $53.64 \pm 9.88$ & $38.64 \pm 6.65$ & $-0.22 *$ & 0.001 & $-15.23 *$ & $<0.0001$ \\
\hline & V5 Gy (\%) & $59.34 \pm 14.39$ & $62.69 \pm 11.54$ & $48.16 \pm 9.81$ & $3.34 *$ & $<0.0001$ & $-11.19 *$ & $<0.0001$ \\
\hline & D1500 (Gy) & $12.17 \pm 5.04$ & $12.18 \pm 4.78$ & $7.37 \pm 3.08$ & $0.02 *$ & 0.002 & $-4.80 *$ & $<0.0001$ \\
\hline & D1000 (Gy) & $19.68 \pm 7.27$ & $20.39 \pm 7.04$ & $14.52 \pm 7.12$ & 0.71 * & 0.012 & $-5.16 *$ & $<0.0001$ \\
\hline
\end{tabular}


Table 4. Cont.

\begin{tabular}{|c|c|c|c|c|c|c|c|c|}
\hline & \multirow{2}{*}{ DV Parameters } & \multirow{2}{*}{$\begin{array}{c}\text { SP-VMAT } \\
\text { Mean } \pm \text { S.D. }\end{array}$} & \multirow{2}{*}{$\begin{array}{c}\text { DP-VMAT } \\
\text { Mean } \pm \text { S.D. }\end{array}$} & \multirow{2}{*}{$\begin{array}{c}\text { H-VMAT } \\
\text { Mean } \pm \text { S.D. }\end{array}$} & \multicolumn{2}{|c|}{$\begin{array}{l}\text { DP-VMAT Minus } \\
\text { SP-VMAT }\end{array}$} & \multicolumn{2}{|c|}{$\begin{array}{l}\text { H-VMAT Minus } \\
\text { SP-VMAT }\end{array}$} \\
\hline & & & & & Diff & $p$ & Diff & $p$ \\
\hline \multirow{6}{*}{$\begin{array}{l}\text { Ipsilateral } \\
\text { Lung }\end{array}$} & Mean Dose (Gy) & $24.46 \pm 5.55$ & $25.64 \pm 5.03$ & $23.88 \pm 5.12$ & $1.18 *$ & 0.002 & -0.58 & 0.058 \\
\hline & V20 Gy (\%) & $52.0 \pm 12.75$ & $53.58 \pm 11.85$ & $45.72 \pm 9.53$ & 1.58 & 0.231 & $-6.82 *$ & $<0.0001$ \\
\hline & V10 Gy (\%) & $63.48 \pm 14.88$ & $69.81 \pm 11.74$ & $59.30 \pm 12.11$ & $6.32 *$ & 0.001 & $-4.18 *$ & 0.002 \\
\hline & V5 Gy (\%) & $61.99 \pm 16.18$ & $69.74 \pm 11.80$ & $59.37 \pm 16.77$ & $7.75 *$ & $<0.0001$ & $-2.62 *$ & 0.004 \\
\hline & D1500 (Gy) & $2.05 \pm 2.61$ & $3.06 \pm 4.10$ & $1.69 \pm 2.09$ & $1.01 *$ & 0.002 & $-0.36 *$ & 0.003 \\
\hline & D1000 (Gy) & $13.23 \pm 10.03$ & $15.85 \pm 9.07$ & $10.26 \pm 8.10$ & $2.61 *$ & 0.012 & $-2.97 *$ & 0.002 \\
\hline \multirow{6}{*}{$\begin{array}{l}\text { Contralateral } \\
\text { Lung }\end{array}$} & Mean Dose (Gy) & $9.41 \pm 2.56$ & $8.71 \pm 1.72$ & $5.82 \pm 1.75$ & $-0.7 *$ & 0.002 & $-3.59 *$ & $<0.0001$ \\
\hline & V20 Gy (\%) & $8.71 \pm 7.54$ & $7.42 \pm 5.18$ & $2.62 \pm 3.13$ & -1.29 & 0.231 & $-6.09 *$ & $<0.0001$ \\
\hline & V10 Gy (\%) & $42.63 \pm 16.45$ & $35.36 \pm 11.69$ & $15.37 \pm 9.97$ & $-7.27 *$ & 0.001 & $-27.27^{*}$ & $<0.0001$ \\
\hline & V5 Gy (\%) & $66.04 \pm 14.36$ & $64.65 \pm 12.59$ & $43.89 \pm 14.38$ & -1.39 & $<0.0001$ & $-22.14 *$ & $<0.0001$ \\
\hline & D1500 (Gy) & $0.78 \pm 1.15$ & $0.688 \pm 1.01$ & $0.54 \pm 0.75$ & $-0.1^{*}$ & 0.002 & $-0.24 *$ & 0.017 \\
\hline & D1000 (Gy) & $5.08 \pm 3.84$ & $4.33 \pm 2.60$ & $5.86 \pm 13.32$ & -0.75 * & 0.012 & $0.77^{*}$ & 0.006 \\
\hline
\end{tabular}

* $p$ value $<0.05$.

\subsection{Dose-Volumetric Parameters of Centrally Located OARs}

DP-VMAT had a similar sparing effect on oesophagus and spinal cord as in SP-VMAT. In DP-VMAT, the heart mean dose was $1.45 \mathrm{~Gy}$ significantly lower than that of SP-VMAT $(p<0.001)$. In view of H-VMAT, oesophagus mean dose, V30 Gy and V18.8 Gy were significantly lower in H-VMAT when compared to SP-VMAT, with 1.73 Gy ( $p=0.02$ ); $5.54 \%,(p=0.003)$ and $7.13 \%,(p=0.002)$ respectively. This also applied to heart V20 Gy and V10 Gy, being 3.34\% $(p=0.02)$ and 7.94\% $(p=0.007)$ lower in H-VMAT than that in SP-VMAT.

However, spinal cord maximum dose and D0.1cc were 5.32 Gy ( $p=0.002)$ and 5.83 Gy $(p=0.02)$ higher in H-VMAT when compared to SP-VMAT respectively. There was significant higher heart maximum dose, V45 Gy, V40 Gy, D0.1cc and D2\% in H-VMAT when compared to SP-VMAT. Details are listed in Table 5.

Table 5. Dose-volumetric parameters of centrally located OARs.

\begin{tabular}{|c|c|c|c|c|c|c|c|c|}
\hline & \multirow{2}{*}{ DV Parameters } & \multirow{2}{*}{$\begin{array}{c}\text { SP-VMAT } \\
\text { Mean } \pm \text { S.D. }\end{array}$} & \multirow{2}{*}{$\begin{array}{c}\text { DP-VMAT } \\
\text { Mean } \pm \text { S.D. }\end{array}$} & \multirow{2}{*}{$\begin{array}{c}\text { H-VMAT } \\
\text { Mean } \pm \text { S.D. }\end{array}$} & \multicolumn{2}{|c|}{$\begin{array}{l}\text { DP-VMAT Minus } \\
\text { SP-VMAT }\end{array}$} & \multicolumn{2}{|c|}{$\begin{array}{l}\text { H-VMAT Minus } \\
\text { SP-VMAT }\end{array}$} \\
\hline & & & & & Diff & $p$ & Diff & $p$ \\
\hline \multirow[t]{4}{*}{ Oesophagus } & Max Dose (Gy) & $59.94 \pm 9.15$ & $60.16 \pm 8.42$ & $59.87 \pm 11.42$ & 0.22 & 0.679 & -0.08 & 0.744 \\
\hline & Mean Dose (Gy) & $24.33 \pm 6.71$ & $24.54 \pm 6.02$ & $22.60 \pm 7.44$ & 0.20 & 0.472 & $-1.73 *$ & 0.02 \\
\hline & V30 Gy (\%) & $39.54 \pm 13.49$ & $37.93 \pm 13.67$ & $33.99 \pm 14.17$ & -1.61 & 0.193 & $-5.54 *$ & 0.003 \\
\hline & V18.8 Gy (\%) & $49.57 \pm 13.53$ & $49.03 \pm 10.62$ & $42.44 \pm 14.80$ & -0.54 & 0.647 & $-7.13 *$ & 0.002 \\
\hline \multirow[t]{2}{*}{ Spinal Cord } & Max Dose (Gy) & $35.87 \pm 7.26$ & $35.3 \pm 7.65$ & $41.19 \pm 3.83$ & -0.14 & 0.679 & $5.32 *$ & 0.002 \\
\hline & $\mathrm{D}_{0.1 \mathrm{cc}}(\mathrm{Gy})$ & $33.42 \pm 7.53$ & $33.4 \pm 7.52$ & $39.29 \pm 3.93$ & -0.06 & 0.811 & $5.83 *$ & 0.02 \\
\hline \multirow[t]{9}{*}{ Heart } & Max Dose (Gy) & $57.85 \pm 16.58$ & $58.87 \pm 14.37$ & $62.51 \pm 7.56$ & 1.02 & 0.102 & $4.67 *$ & 0.002 \\
\hline & Mean Dose (Gy) & $13.66 \pm 8.66$ & $12.20 \pm 8.33$ & $13.74 \pm 8.68$ & $-1.45 *$ & $<0.001$ & 0.08 & 0.879 \\
\hline & V60 Gy (\%) & $2.15 \pm 2.31$ & $2.2 \pm 2.39$ & $2.56 \pm 2.15$ & 0.05 & 0.217 & 0.41 & 0.079 \\
\hline & V45 Gy (\%) & $5.472 \pm 5.21$ & $5.472 \pm 5.1$ & $9.61 \pm 8.23$ & 0.00 & 0.99 & 4.14 * & 0.003 \\
\hline & V40 Gy (\%) & $7.22 \pm 6.56$ & $7.03 \pm 6.61$ & $12.88 \pm 11.21$ & -0.19 & 0.28 & 5.86 * & 0.001 \\
\hline & V20 Gy (\%) & $26.37 \pm 20.63$ & $21.57 \pm 19.03$ & $23.03 \pm 18.06$ & $-4.8 *$ & 0.001 & $-3.34 *$ & 0.02 \\
\hline & V10 Gy (\%) & $47.25 \pm 33.39$ & $40.14 \pm 30.59$ & $39.3 \pm 25.82$ & $-7.11 *$ & $<0.001$ & $-7.94 *$ & 0.007 \\
\hline & $\mathrm{D}_{0.1 \mathrm{cc}}(\mathrm{Gy})$ & $56.94 \pm 16.80$ & $57.8 \pm 14.91$ & $61.48 \pm 8.65$ & 0.86 & 0.094 & $3.68 *$ & 0.001 \\
\hline & $\mathrm{D}_{2 \%}(\mathrm{~Gy})$ & $48.8 \pm 18.17$ & $48.55 \pm 18.66$ & $53.86 \pm 14.29$ & -0.25 & 0.811 & 5.31 * & 0.002 \\
\hline \multirow[t]{3}{*}{ Body } & Mean Dose (Gy) & $8.34 \pm 2.40$ & $8.41 \pm 2.23$ & $7.64 \pm 2.11$ & 0.07 & 0.327 & $-0.70 *$ & $<0.001$ \\
\hline & V10\% (\%) & $32.80 \pm 8.36$ & $33.64 \pm 7.36$ & $25.46 \pm 6.43$ & $0.84 *$ & 0.037 & $-7.34 *$ & $<0.001$ \\
\hline & V30\% (\%) & $16.15 \pm 5.13$ & $15.30 \pm 4.97$ & $13.37 \pm 3.97$ & -0.86 * & 0.001 & $-2.78 *$ & $<0.001$ \\
\hline
\end{tabular}




\section{Discussion}

\subsection{Significance of the Study}

There were no significant differences in the plan quality and dose volumetric parameters in PTV between DP-VMAT and H-VMAT. However, more favourable sparing effects to OARs were found when DP-VMAT and H-VMAT were compared to SP-VMAT.

In H-VMAT, the dose volumetric parameters of lungs (non-PTV lungs, ipsilateral and contralateral lungs) oesophagus and low dose volume of heart (V20 Gy and V10 Gy) were less than those of SP-VMAT, indicated that the sparing effect of these OARs was better when compared to SP-VMAT. For DP-VMAT, there was significant dose reduction to contralateral lung and heart V20 and V10 when compared to SP-VMAT.

\subsection{Lungs Sparing}

Although intensity modulation plans can deliver more homogenous and conformal doses to PTV, when it is applied to lung tumour radiotherapy, large non-PTV lung volume under low dose exposure is a major concern. It is challenging to reduce the non-PTV lung dose when the tumour is centrally located and/or with lymph node involvements. With respect to the clinical trials results of Phase III RTOG 0617, controlling V20 less than $35 \%$ can minimize the risk of pneumonitis [17]. Wijsman et al., 2017 suggested that both acute and late pulmonary toxicity were low in both IMRT and VMAT. However, Shi et al., 2010 study suggested that the severe acute radiation pneumonitis incidence with V10 more than $50 \%$ and V10 less than $50 \%$ were $5.7 \%$ and $29.2 \%$ respectively $(p<0.01)$ [22]. Shaikh el al 2016 studied 139 patients followed up for 5 years. They suggested that V5, V10, V20 and V30 were positively associated with the risk of grade 2 radiation pneumonitis. V5 less than $65 \%$ and V20 less than $25 \%$ were identified as threshold of grade 2 radiation pneumonitis [23]. With the application of immunotherapy (Durvalumab) after chemoirradiation, the constraints were even tightened, with V5 less than 55\%, V20 less than $23 \%$ and mean lung dose less than $14.8 \mathrm{~Gy}$ [24]. In this study, among all lung DV parameters, the non-PTV lung was optimized based on the dose constraints only. The ipsilateral lung, contralateral lung and both lungs may have DV parameters over the dose constraints. In DP-VMAT, dose delivered to contralateral lung was significantly reduced in V10, V5, D1500 and D1000 when compared to SP-VMAT. However, the dose delivered to ipsilateral lung was increased. When considering non-PTV lungs, V5 was significantly increased by $3.45 \%(p=0.006)$, while there was no significant difference in other parameters. The obtained result was expected. For centrally located tumours, both SP-VMAT and DP-VMAT deposited a low dose to ipsilateral and contralateral lung, to achieve a high dose to the PTV. The volume of lung irradiated with a low dose should remain the same in both plans.

In contrast, for H-VMAT, there was significant dose reduction in all DV parameters to non-PTV lung, both lungs, ipsilateral lung and contralateral lungs. In non-PTV lungs, the reduction in volume of $\mathrm{V} 20, \mathrm{~V} 10$ and $\mathrm{V} 5$ was $6.47 \%, 15.89 \%$ and $11.66 \%$ respectively $(p<0.001)$ when compared to SP-VMAT. The reduction of volume was even more for a contralateral lung, with $6.09 \%, 27.27 \%$ and $22.14 \%$ in V20, V10 and V5 respectively. Comparing our H-VMAT results with the abovementioned studies, the probability of grade 2 pneumonitis was much reduced without compromising the dose delivered to PTV [16,21,22]. The effect could be obvious for patients who planned to receive immunotherapy after chemoirradiation. The non-PTV lung dose of our H-VMAT resulted in a V5 of 49.29\% \pm 9.51 ; V20 of $21.83 \% \pm 4.26$ and mean lung dose of $13.19 \mathrm{~Gy} \pm 2.47$, which was lower than the threshold that patients developed pneumonitis at in Landman et al.'s 2021 study [24]. Thus, by controlling the non-PTV lung dose, the progression-free survival would be improved by the application of immunotherapy while limiting the risk of developing pneumonitis.

The major contributor for the V20, V10 and V5 volume reduction was the AP opposing beam arrangement that delivered $50 \%$ of the prescribed dose, the VMAT provided intensity modulation to improve the homogeneity and conformity to the PTV by the rest of 50\% prescribed dose. Hence, the non-PTV lung volume irradiated by low dose could be reduced significantly. Our results are coherent with Mayo et al.'s 2008 study, which illustrated that 
the hybrid of static and intensity modulated beams could better spare the OARs [25]. The benefit was noticeable for contralateral lungs, when a partial arc could be employed to replace the full arc. While in the SP-VMAT and DP-VMAT application, the full arc could not be replaced by partial arc to maintain PTV full coverage and homogeneity.

\subsection{Heart Sparing}

Cardiac injury is a major radiation induced side effect of Hodgkin lymphoma $[26,27]$ and breast cancer $[28,29]$ that developed 10 years after receiving radiotherapy treatment. These patients are young and have favourable prognosis. However, the prognosis for patients with stage III NSCLC is poor, with median survival less than 2 years. In addition, stage III NSCLC patients are generally older and may have more vascular comorbidities and smoking history. The development of cardiac toxicities may be shortened. Wang et al., 2017 reported that patients with mean heart dose received over 20 Gy had a 21\% to develop cardiac events in 2 years [30]. Allan et al., 2015 reported that $8.4 \%$ and $13 \%$ of patients developed cardiac events in 9 and 24 months respectively after they received approximately $63 \mathrm{~Gy}$. Although no association between heart dose and survival has been reported, they suggested to limit the heart V50 and V60 to reduce the rate of cardiac events [31]. All VMAT plans proposed in this study showed very low dose to the heart, even though the heart was near to the PTV in some cases. V20 and V10 had significant reduction in DP-VMAT and H-VMAT when compared to SP-VMAT, suggesting that DP-VMAT and H-VMAT had a better heart sparing effect.

\subsection{Oesophagus Sparing}

Radiation induced oesophagitis has been reported where mean dose, V30 and V60 are the predictor with threshold of $27.5 \mathrm{~Gy}, 43 \%$ and $12.4 \%$, with $\mathrm{V} 60$ is the best among all [16,32]. In our study, V60 was less than 1\% in all three plans, where mean dose and V30 were under the threshold suggested. In particular, significant reduction of mean dose and V30 by 1.73 Gy and $5.54 \%$ was found in H-VMAT when compared to SP-VMAT, suggesting that H-VMAT had good sparing effect on oesophagus.

\subsection{Low Dose Irradiation to Normal Body Tissue}

Although the risk of developing post-radiation sarcoma is rare with a $0.06 \%$ incidence rate, it is associated to the exposure of normal body tissue [33]. Our results demonstrated that the V10 and V30 of normal body tissue received $7.34 \%$ and $2.78 \%$ lower dose in $\mathrm{H}$ VMAT than that in SP-VMAT. Our finding was coherent with Kim et al., 2020 study, that low dose received by normal body tissue could be reduced by including arcs from different planes [11].

\subsection{Duration of Treatment}

For advanced stage NSCLC patients, patient tolerance and treatment reproducibility are our major concerns [16]. With a similar plan quality as IMRT, VMAT is getting popular for its short treatment time. For an SP-VMAT plan, the average treatment time is $4 \mathrm{~min}$ to deliver $2 \mathrm{~Gy}$ in three arcs. One couch kick is required for DP-VMAT, an additional minute is required to move the couch manually; or $20 \mathrm{~s}$ is required to move the couch by dynamic rotation. For H-VMAT, two static fields are included which can be controlled by an automatic field sequencer, and an additional $2 \mathrm{~min}$ is needed when compared to SP-VMAT. In this case, the delivery time remains short in DP-VMAT and H-VMAT. The treatment accuracy and reproducibility can be further improved when breathing motion control could not be offered in our setting.

\subsection{Patient Safety}

For radiotherapy treatment deliver to the thorax, collision is one of the concerns when treatment is delivered by arcs from different planes. The probability of collision increases with couch rotation of more than 30 degrees, large patient size and off-centre tumour 
location [34]. In this study, no couch rotation is employed in SP-VMAT and H-VMAT. For DP-VMAT, 23-30 degree of couch rotation was employed to ensure clearance. In clinical setting, the pre-defined trajectories and immobilization devices must be checked carefully before DP-VMAT plan optimization. Treatment setup trial and moving the couch manually during the first treatment are recommended for DP-VMAT [11].

For DP-VMAT in this study, only a single couch rotation was proposed due to the patient positioning. Full arc delivery from opposite couch rotation was restricted, as the patient was supine with their arms raised over head. For future studies, a partial anterior arc with gantry of 45 to 315 degrees can be adopted with an additional couch kick at opposite direction. It may help to improve the dose reduction in ipsilateral lung and the coverage of PTV if the tumour was located superiorly.

\subsection{Limitations of the Study}

The sample size of was small in this study. This was mainly due to the eligibility criteria of the disease. To minimize the small sample size effect, we used identical CT sets from all patients we collected to produce the SP-VMAT, DP-VMAT and H-VMAT plans to demonstrate the dose volumetric difference was due to the technique difference, but not other factors.

In this study, the Varian 21IX linear accelerator was selected based on the real clinical setting in PWH. It was not equipped with jaw tracking. Previous studies demonstrated that jaw tracking technique reduced the dose to an ipsilateral lung VMAT in locally advanced NSCLC without jeopardising the conformity and homogeneity to PTV. In addition, there was significant dose reduction of V20 in contralateral lung, V35 in the oesophagus and other OARs $[35,36]$. With the jaw tracking capability, radiation dose to OARs might be further reduced in SP-VMAT, DP-VMAT and H-VMAT. Further investigation is suggested in future study.

For DP-VMAT, \pm 23 to 30 degree couch rotation was chosen in the current study to demonstrate the effect on OARs sparing. Other couch and gantry configurations can be adopted in future studies to evaluate which settings could achieve better OARs sparing effects for patients with NSCLC.

About the Weighting of 3DCRT/VMAT in H-VMAT plan, 50\%:50\% of prescribed dose was allocated in 3DCRT and VMAT components. Other combinations including $40 \%: 60 \% ; 60 \%: 40 \%$ and 30\%:70\% were planned for the selected case as a trial for plan quality evaluation. Mayo et al. reported that using a two-thirds dose in static fields and a one-third dose in intensity modulated beams improved OARs sparing capabilities [25]. Our findings were comparable with the results from Chan et al. in 2011 that two partial arcs plus two static fields with $50 \%$ in each of the component yielded a better conformity to PTV and reduced the dose to non-PTV lung [37]. Other combinations showed either less reduction in volume to non-PTV lungs (less weighting in 3DCRT, i.e., $40 \%: 60 \%$ and $30 \%: 70 \%)$, reduced in conformity to PTV (30\%:70\%) or higher dose to the heart (60\%:40\%). Based on the trial findings, the optimal weighting of 50\%:50\% was chosen for the H-VMAT plan in this study.

Other than modifying treatment plans (from SP to DP or H-VMAT) before treatment starts, adaptive radiotherapy is an approach which focuses on re-planning during treatment course, to improve the therapeutic ratio and minimise the dose to OARs [38]. The application was essential for patients with substantial tumour shrinkage and geometrical uncertainties during the treatment course [39]. The dose reduction demonstrated in this study will be further enhanced by incorporating it with adaptive radiotherapy.

\section{Conclusions}

The proposed DP-VMAT and H-VMAT showed similar HI, CI and PTV dose volumetric, but significant dose reductions to OARs when compared with the SP-VMAT for centrally located stage III NSCLC. The proposed H-VMAT showed favourable plan quality especially to non-PTV lungs and the oesophagus. In particular, the H-VMAT plan could be 
considered for patients who were planned for immunotherapy after standard treatment. The DP-VMAT plan showed significant dose reduction to the heart when compared to the H-VMAT plan. Therefore, modified VMAT proposed in this study can be considered in a clinical setting, to develop a new standard of care for locally advanced centrally located NSCLC patients that pulmonary, cardiac, and oesophageal functions can be preserved after radiotherapy.

Author Contributions: Conceptualization, E.Y.W.C., K.T.K.C., K.T.Y.C., T.S.W.C. and J.C.L.Y.; methodology, E.Y.W.C., K.T.K.C., K.T.Y.C., T.S.W.C. and J.C.L.Y.; software, E.Y.W.C., K.T.K.C., K.T.Y.C., T.S.W.C. and J.C.L.Y.; validation, E.Y.W.C., F.Y.C.C., D.Y.T.C., J.K.Y.C. and S.H.Y.Y.; formal analysis, E.Y.W.C., F.Y.C.C., D.Y.T.C., J.K.Y.C. and S.H.Y.Y.; investigation, E.Y.W.C., K.T.K.C., K.T.Y.C., T.S.W.C. and J.C.L.Y.; data curation, E.Y.W.C., V.H.Y.K., K.T.K.C., K.T.Y.C., T.S.W.C. and J.C.L.Y.; writingoriginal draft preparation, E.Y.W.C.; writing-review and editing, E.Y.W.C.; supervision, E.Y.W.C. and V.H.Y.K.; project administration, E.Y.W.C. and V.H.Y.K.; All authors have read and agreed to the published version of the manuscript.

Funding: This research received no external funding.

Institutional Review Board Statement: The study was conducted according to the guidelines of the Declaration of Helsinki, and approved by the Research Ethics Committee of the Hospital Authority, The New Territories East Cluster, Hong Kong SAR (CREC Ref No: CUHK-NTEC 2019.655).

Informed Consent Statement: Patient consent was waived, due to the study's retrospective nature. Patient records, information and images were anonymized prior to analysis.

Data Availability Statement: The clinical and MRI data are not publicly available for patient privacy protection purposes.

Acknowledgments: This study was supported by Tung Wah College and the Clinical Oncology Department of the Prince of Wales Hospital Hong Kong.

Conflicts of Interest: The authors declare no conflict of interest.

\section{References}

1. Sung, H.; Ferlay, J.; Siegel, R.L.; Laversanne, M.; Soerjomataram, I.; Jemal, A.; Bray, F. Global Cancer Statistics 2020: GLOBOCAN Estimates of Incidence and Mortality Worldwide for 36 Cancers in 185 Countries. CA A Cancer J. Clin. 2021, 71, 209-249. [CrossRef] [PubMed]

2. Hong Kong Cancer Registry, Hospital Authority. Available online: https://www3.ha.org.hk/cancereg/topten.html (accessed on 3 August 2021).

3. Ettinger, D.S.; Wood, D.E.; Akerley, W.; Bazhenova, L.A.; Borghaei, H.; Camidge, D.R.; Cheney, R.T.; Chirieac, L.R.; D'Amico, T.A.; Dilling, T.J.; et al. NCCN Guidelines Insights: Non-Small Cell Lung Cancer, Version 4.2016. J. Natl. Compr. Cancer Netw. 2016, 14, 255-264. [CrossRef]

4. Bradley, J.D.; Hu, C.; Komaki, R.R.; Masters, G.A.; Blumenschein, G.R.; Schild, S.E.; Bogart, J.A.; Forster, K.M.; Magliocco, A.M.; Kavadi, V.S.; et al. Long-Term Results of NRG Oncology RTOG 0617: Standard- Versus High-Dose Chemoradiotherapy With or Without Cetuximab for Unresectable Stage III Non-Small-Cell Lung Cancer. J. Clin. Oncol. 2020, 38, 706-714. [CrossRef] [PubMed]

5. Jia, B.; Zheng, Q.; Qi, X.; Zhao, J.; Wu, M.; An, T.; Wang, Y.; Zhuo, M.; Li, J.; Zhao, X.; et al. Survival comparison of right and left side non-small cell lung cancer in stage I-IIIA patients: A Surveillance Epidemiology and End Results (SEER) analysis. Thorac. Cancer 2019, 10, 459-471. [CrossRef]

6. Palma, D.A.; Senan, S.; Oberije, C.; Belderbos, J.; DE Dios, N.R.; Bradley, J.D.; Barriger, R.B.; Moreno-Jiménez, M.; Kim, T.H.; Ramella, S.; et al. Predicting Esophagitis After Chemoradiation Therapy for Non-Small Cell Lung Cancer: An Individual Patient Data Meta-Analysis. Int. J. Radiat. Oncol. Biol. Phys. 2013, 87, 690-696. [CrossRef]

7. Hernando, M.L.; Marks, L.B.; Bentel, G.C.; Zhou, S.-M.; Hollis, D.; Das, S.K.; Fan, M.; Munley, M.T.; Shafman, T.D.; Anscher, M.; et al. Radiation-induced pulmonary toxicity: A dose-volume histogram analysis in 201 patients with lung cancer. Int. J. Radiat. Oncol. Biol. Phys. 2001, 51, 650-659. [CrossRef]

8. Aupérin, A.; Le Péchoux, C.; Rolland, E.; Curran, W.J.; Furuse, K.; Fournel, P.; Belderbos, J.; Clamon, G.; Ulutin, H.C.; Paulus, R.; et al. Meta-Analysis of Concomitant Versus Sequential Radiochemotherapy in Locally Advanced Non-Small-Cell Lung Cancer. JCO 2010, 28, 2181-2190. [CrossRef]

9. Ahn, J.S.; Ahn, Y.C.; Kim, J.-H.; Lee, C.G.; Cho, E.K.; Lee, K.C.; Chen, M.; Kim, D.-W.; Kim, H.-K.; Min, Y.J.; et al. Multinational Randomized Phase III Trial with or Without Consolidation Chemotherapy Using Docetaxel and Cisplatin After Concurrent Chemoradiation in Inoperable Stage III Non-Small-Cell Lung Cancer: KCSG-LU05-04. JCO 2015, 33, 2660-2666. [CrossRef] 
10. Antonia, S.J.; Villegas, A.; Daniel, D.; Vicente, D.; Murakami, S.; Hui, R.; Yokoi, T.; Chiappori, A.; Lee, K.H.; De Wit, M.; et al. Durvalumab after Chemoradiotherapy in Stage III Non-Small-Cell Lung Cancer. N. Engl. J. Med. 2017, 377, 1919-1929. [CrossRef]

11. Kim, S.-T.; An, H.J.; Kim, J.; Yoo, J.-R.; Kim, H.J.; Park, J.M. Non-coplanar VMAT plans for lung SABR to reduce dose to the heart: A planning study. BJR 2020, 93, 20190596. [CrossRef]

12. Ma, M.; Ren, W.; Li, M.; Niu, C.; Dai, J. Dosimetric comparison of coplanar and noncoplanar beam arrangements for radiotherapy of patients with lung cancer: A meta-analysis. J. Appl. Clin. Med. Phys. 2021, 22, 34-43. [CrossRef]

13. Govaert, S.L.; Troost, E.G.; Schuurbiers, O.C.; de Geus-Oei, L.-F.; Termeer, A.; Span, P.N.; Bussink, J. Treatment outcome and toxicity of intensity-modulated (chemo) radiotherapy in stage III non-small cell lung cancer patients. Radiat. Oncol. 2012, 7, 150. [CrossRef] [PubMed]

14. Cheung, E.Y.W.; Lee, K.H.Y.; Lau, W.T.L.; Lau, A.P.; Wat, P.Y. Non-coplanar VMAT plans for postoperative primary brain tumour to reduce dose to hippocampus, temporal lobe and cochlea: A planning study. BJR Open 2021, 3, 20210009. [CrossRef]

15. Song, C.H.; Pyo, H.; Moon, S.H.; Kim, T.H.; Kim, D.W.; Cho, K.H. Treatment-Related Pneumonitis and Acute Esophagitis in Non-Small-Cell Lung Cancer Patients Treated with Chemotherapy and Helical Tomotherapy. Int. J. Radiat. Oncol. Biol. Phys. 2010, 78, 651-658. [CrossRef] [PubMed]

16. Steber, J.L.; Silva, S.; Harkenrider, M.M.; Surucu, M.; Choi, M. Clinical Application of a Hybrid Volumetric Arc Therapy Technique for Locally Advanced Lung Cancer. Int. J. Radiat. Oncol. Biol. Phys. 2015, 93, E595. [CrossRef]

17. Bradley, J.D.; Paulus, R.; Komaki, R.; Masters, G.; Blumenschein, G.; Schild, S.; Bogart, J.; Hu, C.; Forster, K.; Magliocco, A.; et al. Standard-dose versus high-dose conformal radiotherapy with concurrent and consolidation carboplatin plus paclitaxel with or without cetuximab for patients with stage IIIA or IIIB non-small-cell lung cancer (RTOG 0617): A randomised, two-by-two factorial phase 3 study. Lancet Oncol. 2015, 16, 187-199. [CrossRef]

18. Nelms, B.E.; Robinson, G.; Markham, J.; Velasco, K.; Boyd, S.; Narayan, S.; Wheeler, J.; Sobczak, M.L. Variation in external beam treatment plan quality: An inter-institutional study of planners and planning systems. Pract. Radiat. Oncol. 2012, 2, $296-305$. [CrossRef]

19. Kataria, T.; Sharma, K.; Subramani, V.; Karrthick, K.; Bisht, S. Homogeneity Index: An objective tool for assessment of conformal radiation treatments. J. Med. Phys. 2012, 37, 207. [CrossRef]

20. Landberg, T.; Chavaudra, J.; Dobbs, J.; Gerard, J.-P.; Hanks, G.; Horiot, J.-C.; Johansson, K.-A.; Möller, T.; Purdy, J.; Suntharalingam, N.; et al. Report 62. J. Int. Comm. Radiat. Units Meas. 1999, os32, NP. [CrossRef]

21. Greenland, S.; Senn, S.J.; Rothman, K.J.; Carlin, J.B.; Poole, C.; Goodman, S.N.; Altman, D.G. Statistical tests, P values, confidence intervals, and power: A guide to misinterpretations. Eur. J. Epidemiol. 2016, 31, 337-350. [CrossRef]

22. Shi, A.; Zhu, G.; Wu, H.; Yu, R.; Li, F.; Xu, B. Analysis of clinical and dosimetric factors associated with severe acute radiation pneumonitis in patients with locally advanced non-small cell lung cancer treated with concurrent chemotherapy and intensitymodulated radiotherapy. Radiat. Oncol. 2010, 5, 35. [CrossRef]

23. Shaikh, T.; Churilla, T.M.; Monpara, P.; Scott, W.J.; Cohen, S.J.; Meyer, J.E. Risk of radiation pneumonitis in patients receiving taxane-based trimodality therapy for locally advanced esophageal cancer. Pr. Radiat. Oncol. 2016, 6, 388-394. [CrossRef]

24. Landman, Y.; Jacobi, O.; Kurman, N.; Yariv, O.; Peretz, I.; Rotem, O.; Dudnik, E.; Zer, A.; Allen, A.M. Durvalumab after concurrent chemotherapy and high-dose radiotherapy for locally advanced non-small cell lung cancer. OncoImmunology 2021, 10, 1959979. [CrossRef] [PubMed]

25. Mayo, C.S.; Urie, M.M.; Fitzgerald, T.J.; Ding, L.; Lo, Y.C.; Bogdanov, M. Hybrid IMRT for Treatment of Cancers of the Lung and Esophagus. Int. J. Radiat. Oncol. Biol. Phys. 2008, 71, 1408-1418. [CrossRef] [PubMed]

26. van Nimwegen, F.A.; Schaapveld, M.; Janus, C.P.M.; Krol, S.; Petersen, E.J.; Raemaekers, J.M.M.; Kok, W.E.M.; Aleman, B.M.P.; Van Leeuwen, F.E. Cardiovascular disease after Hodgkin lymphoma treatment: 40-year disease risk. JAMA Intern. Med. 2015, 175, 1007-1017. [CrossRef] [PubMed]

27. van Nimwegen, F.A.; Schaapveld, M.; Cutter, D.J.; Janus, C.P.; Krol, A.D.; Hauptmann, M.; Kooijman, K.; Roesink, J.; van der Maazen, R.; Darby, S.C.; et al. Radiation Dose-Response Relationship for Risk of Coronary Heart Disease in Survivors of Hodgkin Lymphoma. J. Clin. Oncol. 2016, 34, 235-243. [CrossRef]

28. Hooning, M.J.; Botma, A.; Aleman, B.M.P.; Baaijens, M.H.A.; Bartelink, H.; Klijn, J.G.M.; Taylor, C.W.; Van Leeuwen, F.E. Long-term risk of cardiovascular disease in 10-year survivors of breast cancer. J. Natl. Cancer Inst. 2007, 99, 365-375. [CrossRef]

29. Darby, S.C.; McGale, P.; Taylor, C.W.; Peto, R. Long-term mortality from heart disease and lung cancer after radiotherapy for early breast cancer: Prospective cohort study of about 300,000 women in US SEER cancer registries. Lancet Oncol. 2005, 6, 557-565. [CrossRef]

30. Wang, K.; Eblan, M.J.; Deal, A.M.; Lipner, M.; Zagar, T.M.; Wang, Y.; Mavroidis, P.; Lee, C.B.; Jensen, B.C.; Rosenman, J.G.; et al. Cardiac Toxicity After Radiotherapy for Stage III Non-Small-Cell Lung Cancer: Pooled Analysis of Dose-Escalation Trials Delivering 70 to 90 Gy. JCO 2017, 35, 1387-1394. [CrossRef]

31. Allan, E.; Williams, T.M.; Grecula, J.C.; Haglund, K.E.; Welliver, M.X.; Bazan, J.G. Mortality, Cardiac Toxicity, and Radiation Dose to the Heart in Patients Treated with Curative Intent Fractionated Radiation Therapy for Lung Cancer. Int. J. Radiat. Oncol. Biol. Phys. 2015, 93, S161. [CrossRef]

32. Bourbonne, V.; Delafoy, A.; Lucia, F.; Quéré, G.; Pradier, O.; Schick, U. Toxicity after volumetric modulated arc therapy for lung cancer: A monocentric retrospective study. Transl. Lung Cancer Res. 2021, 10, 156-166. [CrossRef] [PubMed] 
33. Mavrogenis, A.F.; Pala, E.; Guerra, G.; Ruggieri, P. Post-radiation sarcomas. Clinical outcome of 52 Patients. J. Surg Oncol. 2012, 105, 570-576. [CrossRef]

34. Smyth, G.; Evans, P.M.; Bamber, J.C.; Bedford, J.L. Recent developments in non-coplanar radiotherapy. BJR 2019, 92, 20180908. [CrossRef] [PubMed]

35. Lee, M.F.; Wang, M.S.; Lai, Y.H.; Chen, H.H.W. Comparison of Dosimetry and Efficiency of Jaw Tracking and Static Jaw Techniques Using Volumetric Modulated Arc Therapy and Intensity-Modulated Radiation Therapy in Non-Small Cell Lung Cancer. Int. J. Radiat. Oncol. Biol. Phys. 2018, 102, e496. [CrossRef]

36. Pokhrel, D.; Sanford, L.; Halfman, M.; Molloy, J. Potential reduction of lung dose via VMAT with jaw tracking in the treatment of single-isocenter/two-lesion lung SBRT. J. Appl. Clin. Med. Phys. 2019, 20, 55-63. [CrossRef] [PubMed]

37. Chan, O.S.H.; Lee, M.C.H.; Hung, A.W.M.; Chang, A.T.Y.; Yeung, R.M.W.; Lee, A.W.M. The superiority of hybrid-volumetric arc therapy (VMAT) technique over double arcs VMAT and 3D-conformal technique in the treatment of locally advanced non-small cell lung cancer-A planning study. Radiother. Oncol. 2011, 101, 298-302. [CrossRef]

38. Kataria, T.; Gupta, D.; Bisht, S.S.; Karthikeyan, N.; Goyal, S.; Pushpan, L.; Abhishek, A.; Govardhan, H.; Kumar, V.; Sharma, K.; et al. Adaptive radiotherapy in lung cancer: Dosimetric benefits and clinical outcome. BJR 2014, 87, 20130643. [CrossRef]

39. Woodford, C.; Yartsev, S.; Dar, A.R.; Bauman, G.; van Dyk, J. Adaptive Radiotherapy Planning on Decreasing Gross Tumor Volumes as Seen on Megavoltage Computed Tomography Images. Int. J. Radiat. Oncol. Biol. Phys. 2007, 69, 1316-1322. [CrossRef] 